\title{
Modeling of amorphous InGaZnO 4 thin film transistors and their subgap density of states
}

\author{
Hsing-Hung Hsieh, ${ }^{1,2}$ Toshio Kamiya, ${ }^{3,4}$ Kenji Nomura, ${ }^{4}$ Hideo Hosono, ${ }^{1,3,4, a)}$ and \\ Chung-Chih $\mathrm{Wu}^{2,5,6}$ \\ ${ }^{1}$ Frontier Collaborative Research Center, Tokyo Institute of Technology, 4259 Nagatsuta, Midori-ku, \\ Yokohama 226-8503, Japan \\ ${ }^{2}$ Graduate Institute of Electronics Engineering, National Taiwan University, Taipei 10617, Taiwan \\ ${ }^{3}$ Materials and Structures Laboratory, Tokyo Institute of Technology, 4259 Nagatsuta, Midori-ku, \\ Yokohama 226-8503, Japan \\ ${ }^{4}$ ERATO-SORST, Japan Science and Technology Agency, in Frontier Collaborative Research Center, \\ Tokyo Institute of Technology, 4259 Nagatsuda, Midori-ku, Yokohama 226-8503, Japan \\ ${ }^{5}$ Graduate Institute of Photonics and Optoelectronics, National Taiwan University, Taipei 10617, Taiwan \\ ${ }^{6}$ Department of Electrical Engineering, National Taiwan University, Taipei 10617, Taiwan
}

(Received 26 November 2007; accepted 24 January 2008; published online 1 April 2008)

\begin{abstract}
We report a model of the carrier transport and the subgap density of states in amorphous $\mathrm{InGaZnO}_{4}$ ( $a$-IGZO) for device simulation of $a$-IGZO thin-film transistors (TFTs) operated in both the depletion mode and the enhancement mode. A simple model using a constant mobility and two-step subgap density of states reproduced well the characteristics of the $a$-IGZO TFTs. $a$-IGZO exhibits low densities of tail states and deep gap states, leading to small subthreshold swings and high mobilities. (C) 2008 American Institute of Physics. [DOI: 10.1063/1.2857463]
\end{abstract}

Amorphous semiconductors have been widely pursued for decades due to their wide applications and high uniformity. $^{1-3}$ Recently, amorphous oxide semiconductors (AOSs) have attracted much attention because AOS thin-film transistors (TFTs) exhibit large mobilities with lowtemperature or even room-temperature fabrication. ${ }^{4-9}$ The conduction bands of the AOSs are derived from the $n s$ orbitals of heavy metal cations such as $\mathrm{In}^{3+}, \mathrm{Ga}^{3+}$, and $\mathrm{Zn}^{2+}$. The electron transport path is very efficient because of the large radii and large overlap between adjacent $n s$ orbitals of spherical symmetry, which is less sensitive to the arrangement of atoms. As such, oxide semiconductors can show comparable mobilities both in crystalline and amorphous phases, while covalent semiconductors such as silicon show at least two to three orders of magnitude smaller mobilities in the amorphous phase.

Despite the rapid advance of the AOS device technology, many physical properties of AOSs are still not well understood. Although the fundamental transport properties and electronic structures of AOSs had been studied, ${ }^{10,11}$ the subgap density of states (DOSs), which is also essential for the noncrystalline materials, ${ }^{12-14}$ have not yet been reported. In this letter, we report a model of electron transport and subgap DOSs in a representative AOS, amorphous $\mathrm{InGaZnO}_{4}(a$-IGZO), based on TFT device simulations using TCAD. ${ }^{15}$ We employed a standard model developed for silicon technology, which considers parameterized subgap DOSs.

The $a$-IGZO TFTs studied (inset of Fig. 3) were fabricated on a heavily doped silicon substrate (used as the gate as well) with a $100-\mathrm{nm}$ thermal $\mathrm{SiO}_{2}$ as the gate insulator. The $a$-IGZO channels $(40 \mathrm{~nm})$ were deposited at room temperature by pulsed laser deposition (PLD), and then annealed at $300{ }^{\circ} \mathrm{C}$ in air for $1 \mathrm{~h}$. The IGZO films remained amorphous after $300{ }^{\circ} \mathrm{C}$ annealing. ${ }^{16}$ Finally, $30 \mathrm{~nm}$ indium tin

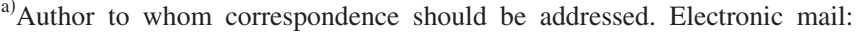
hosono@msl.titech.ac.jp. oxide (ITO) was deposited at room temperature by PLD and then patterned by the lift-off process to form source and drain electrodes. The channel width and length of the TFTs were 300 and $50 \mu \mathrm{m}$, respectively. The electron concentrations in $a$-IGZO films were controlled by the oxygen partial pressure $\left(P_{\mathrm{O}_{2}}\right)$ during the PLD process. A lower $P_{\mathrm{O}_{2}}$ of $5.2 \mathrm{~Pa}$ resulted in a larger electron concentration of $\sim 4$ $\times 10^{17} \mathrm{~cm}^{-3}$ (Ref. 16) and the depletion-type TFTs, while a higher $P_{\mathrm{O}_{2}}$ of $6.5 \mathrm{~Pa}$ resulted in a lower electron concentration of $\sim 1 \times 10^{15} \mathrm{~cm}^{-3}$ and the enhancement-type TFTs. The typical measured transfer and output characteristics of both types of devices are shown in Fig. 1. The saturation electron
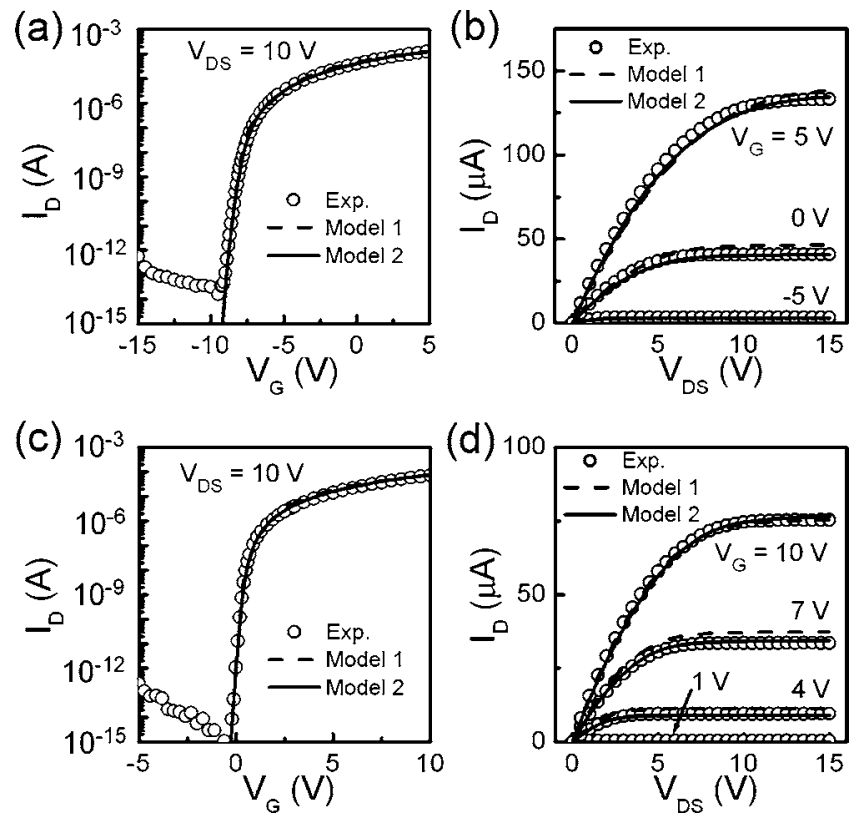

FIG. 1. (a) Measured transfer characteristics and (b) output characteristics of the depletion-type TFT and (c) measured transfer characteristics and (d) output characteristics of the enhancement-type TFT along with optimized fittings using model 1 (dashed lines) and model 2 (solid lines). $I_{D}$ is the drain current and circles represent the experimental data. 
mobilities and threshold voltages extracted with standard current equation of field-effect transistors are $9.19 \mathrm{~cm}^{2}(\mathrm{~V} \mathrm{~s})^{-1}$ and $-6.54 \mathrm{~V}$ for the depletion-type TFT, and $7.84 \mathrm{~cm}^{2}(\mathrm{~V} \mathrm{~s})^{-1}$ and $0.49 \mathrm{~V}$ for the enhancement-type TFT, respectively.

A device simulator ATLAS (Silvaco) was used to simulate TFT characteristics and to extract subgap DOSs. ATLAS treats the subgap DOSs with four parameterized components, the acceptor-like exponential function, the acceptor-like Gaussian function, the donor-like exponential function, and the donor-like Gaussian function. Considering that $a$-IGZO is a $n$-type semiconductor and that $a$-IGZO TFTs operate in the $n$-channel mode, we considered mainly the acceptor-like states. The acceptor-like exponential DOS is described by

$$
g_{\exp }(E)=N_{\mathrm{TA}} \cdot \exp \left[\frac{-\left(E_{C}-E\right)}{W_{\mathrm{TA}}}\right],
$$

where $E$ is the state energy, $E_{C}$ is the conduction band edge, $N_{\mathrm{TA}}$ is the intercept density at $E_{C}$, and $W_{\mathrm{TA}}$ is the characteristic decay energy. The acceptor-like Gaussian DOS is described by

$$
g_{G}(E)=N_{\mathrm{GA}} \cdot \exp \left[-\left(\frac{E_{0}-E}{W_{\mathrm{GA}}}\right)^{2}\right],
$$

where $N_{\mathrm{GA}}$ is the density at the central energy $E_{0}$ of the Gaussian distribution, and $W_{\mathrm{GA}}$ is the characteristic decay energy. For hydrogenated amorphous silicon $(a-\mathrm{Si}: \mathrm{H})$, exponential DOSs are used for the tail states near the conduction and valence band edges and Gaussian DOSs are used for the deep gap states. After preliminary simulations, we found that the same assignments were also valid for $a$-IGZO, except that the deep gap states exhibiting clear peak structures within the band gap did not reproduce well the characteristics of the $a$-IGZO TFTs. Therefore, $E_{0}$ was fixed at $E_{C}$ to represent a monotonously decreasing deep gap states, while $N_{\mathrm{TA}}, W_{\mathrm{TA}}, N_{\mathrm{GA}}$, and $W_{\mathrm{GA}}$ were determined by fitting to the experimental data. When all these four parameters are optimized in the fitting processes, the electron mobility $\left(\mu_{n}\right)$, the electron concentration $\left(n_{0}\right)$ of $a$-IGZO, and the difference between the work function of ITO and the electron affinity of $a$-IGZO $(\Delta \phi)$ may be fine adjusted to further improve the fitting.

Figures 1(a) and 1(b) show the optimized fittings to the experimental transfer and output characteristics of a typical depletion-type $a$-IGZO TFT, using two different DOS models. Model 1 considered tail states only, while model 2 incorporated both tail states and deep gap states. The optimized fitting parameters are listed in Table I. Although both models appear to reproduce well the transfer characteristics, as

TABLE I. Optimized fitting parameters for the depletion-type and the enhancement-type $a$-IGZO TFTs using models 1 and 2 .

\begin{tabular}{lccccc}
\hline \hline & \multicolumn{2}{c}{ Depletion type } & & \multicolumn{2}{c}{ Enhancement type } \\
\cline { 2 - 3 } \cline { 6 - 6 } & Model 1 & Model 2 & & Model 1 & Model 2 \\
\hline$N_{T A}\left(\mathrm{~cm}^{-3} \mathrm{eV}^{-1}\right)$ & $4.5 \times 10^{17}$ & $2.3 \times 10^{18}$ & & $5.0 \times 10^{16}$ & $2.3 \times 10^{18}$ \\
$W_{T A}(\mathrm{eV})$ & 0.35 & 0.14 & & 1.0 & 0.08 \\
$N_{G A}\left(\mathrm{~cm}^{-3} \mathrm{eV}^{-1}\right)$ & $\ldots$ & $4.5 \times 10^{16}$ & & $\cdots$ & $3.2 \times 10^{16}$ \\
$W_{G A}(\mathrm{eV})$ & $\ldots$ & 1.5 & & $\ldots$ & 1.5 \\
$\mu_{\mathrm{n}}\left[\mathrm{cm}^{2}(\mathrm{~V} \mathrm{~s})^{-1}\right]$ & 9.63 & 11.88 & & 9.10 & 8.57 \\
$\mathrm{n}_{0}\left(\mathrm{~cm}^{-3}\right)$ & $4.0 \times 10^{17}$ & $4.1 \times 10^{17}$ & & $1 \times 10^{15}$ & $1 \times 10^{15}$ \\
$\Delta \phi(\mathrm{eV})$ & 0.21 & 0.16 & & 0.33 & 0.20 \\
\hline \hline
\end{tabular}
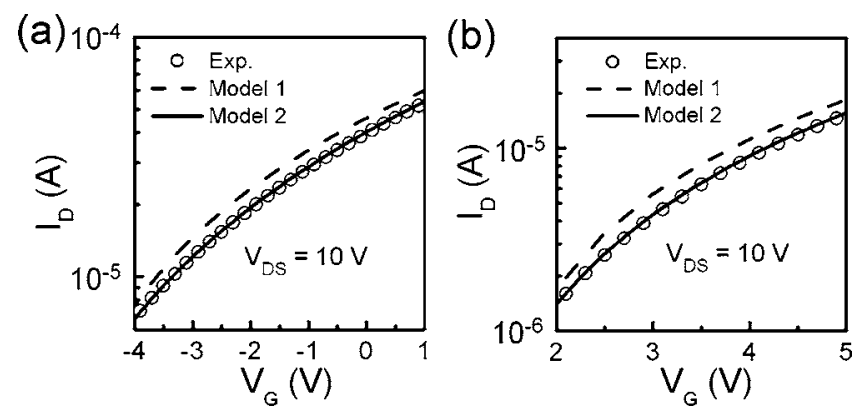

FIG. 2. Enlargements of the transfer characteristics of (a) depletion-type and (b) enhancement-type TFTs, showing non-negligible deviations from measurements when model 1 was used.

shown in Fig. 1(a), the fitting using model 1 (dashed line) shows nonnegligible deviations especially in the accumulation region [gate voltage $V_{G}>-4 \mathrm{~V}$, Fig. 2(a)]. For the output characteristics [Fig. 1(b)], while model 2 gives nice fittings to measured curves, it is generally difficult to get universally good fittings using model 1 for extended combinations of $V_{G}$ (gate voltage) and $V_{\mathrm{DS}}$ (drain-to-source voltage) and obvious deviations are seen in the saturation regime. Similar situations also occurred in the enhancementtype TFTs [Figs. 1(c), 1(d), and 2(b)]. Thus, incorporation of both tail states and deep gap states is necessary to accurately reproduce characteristics of $a$-IGZO TFTs.

Using DOS fitting parameters listed in Table I for model 2, Fig. 3 illustrates the corresponding subgap DOSs for both TFTs. Since the conduction band minimum (CBM) of $a$-IGZO is primarily composed of In $5 s$ orbitals, ${ }^{11}$ the continuous tail states extending from the CBM originate from variation of In-O-metal bonding angles. The concrete entity of the deep gap states in $a$-IGZO (and other AOSs) is yet to be determined, but oxygen deficiency play some roles speculated from analogy to crystalline oxide semiconductors. Despite these uncertainties, the two-step DOS is a simple and general model. Since the Fermi level in the channel varies in the range from $E_{C}$ to $E_{C}-0.8 \mathrm{eV}$ for the $V_{\mathrm{DS}}$ and $V_{G}$ examined here, the extracted DOSs have physical validity only in this energy range (i.e., the solid line region). The subgap DOS is slightly larger in the depletion-type TFT than in the enhancement-type TFT, since the $a$-IGZO channel deposited at a lower $P_{\mathrm{O}_{2}}$ would contain more oxygen deficiencies. $N_{\mathrm{TA}}$ in $a-\mathrm{Si}: \mathrm{H}$ is typically more than $10^{21}$ or $10^{22} \mathrm{~cm}^{-3} \mathrm{eV}^{-1}$, ${ }^{12}$ which is two to three orders of magnitude larger than that of $a$-IGZO. Due to low subgap DOS in

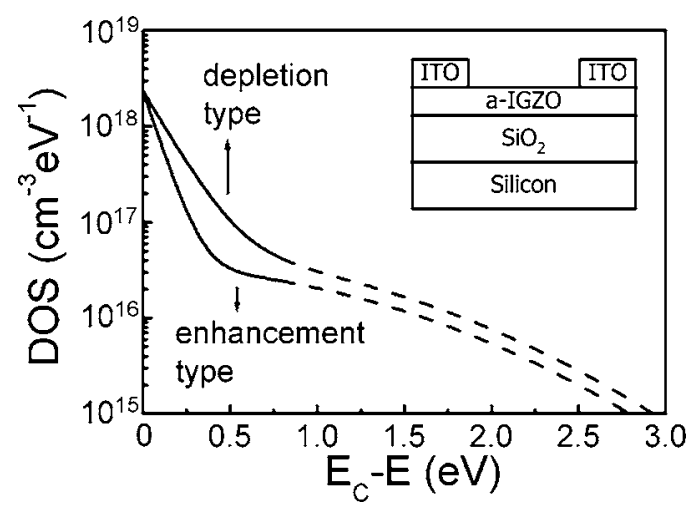

FIG. 3. The subgap DOSs for the depletion-type and enhancement-type TFTs extracted using model 2 . The extracted DOSs have physical validity only in the solid line region. 

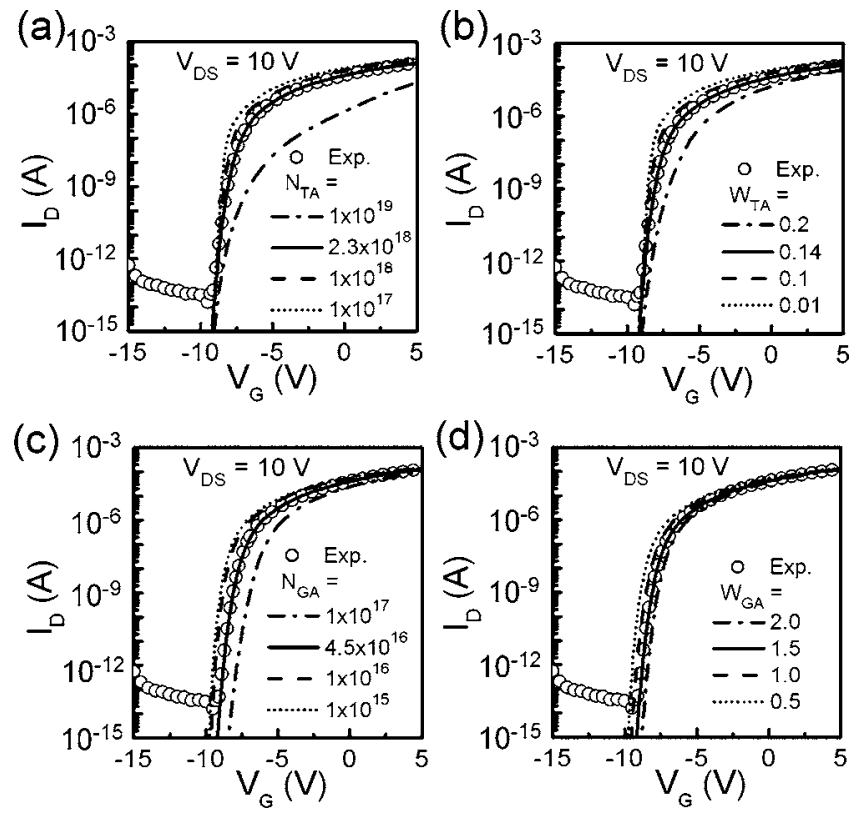

FIG. 4. Influences of (a) $N_{\mathrm{TA}}$, (b) $W_{\mathrm{TA}}$, (c) $N_{\mathrm{GA}}$, and (d) $W_{\mathrm{GA}}$ on transfer characteristics of the $a$-IGZO TFT. Parameters other than those indicated in the figures are fixed to the values in Table I (depletion-type TFT, model 2).

AOSs, it shall be much easier to move the Fermi level toward the mobility edge and attain bandlike conduction, explaining high mobilities in AOSs.

To probe the reliability of the modeling and the influences of each subgap DOS parameter $\left(N_{\mathrm{TA}}, W_{\mathrm{TA}}, N_{\mathrm{GA}}\right.$, and $\left.W_{\mathrm{GA}}\right)$ on device characteristics, the transfer characteristics of the $a$-IGZO TFT were simulated (Fig. 4). In each panel of Fig. 4, only one subgap DOS parameter was varied at a time, while the other fitting parameters remained the same as those listed in Table I (depletion-type TFT, model 2). One sees that tail states have stronger effects on the subthreshold swing $(S)$ and the on current. $S$ rises and the on current (at a gate voltage) drops as tail states increase. Meanwhile, the turn-on voltage (simply defined as $V_{G}$ at which $I_{D}$ starts to rise sharply in the transfer curve) remains rather intact in varying of tail states. On the contrary, the variations of the deep gap states (i.e., $N_{\mathrm{GA}}$ and $W_{\mathrm{GA}}$ ) have the major effect of shifting the turn-on voltage and the transfer curve, while the subthreshold swing and on current stay nearly unchanged. These statements are particularly true as $N_{\mathrm{GA}}$ is significantly lower than $N_{\mathrm{TA}}$, as in the present case of modeling $a$-IGZO TFTs. As such, the subgap DOS is dominated by the exponential tails states near the conduction band and by the Gaussiantype states deeper into the band gap. Figures 3 and 4 together indicate that the Fermi level moves from deep gap toward $E_{C}$ across the kink region of the subgap DOS as the device operation is switched from the off state to the subthreshold state, lending control of the turn-on voltages to deep gap states $\left(N_{\mathrm{GA}}\right.$ and $\left.W_{\mathrm{GA}}\right)$ while the subthreshold and threshold behaviors as well as on state currents to tail states $\left(N_{\mathrm{TA}}\right.$ and $\left.W_{\mathrm{TA}}\right)$. The present model focuses on the on-state and subthreshold characteristics. The off-state currents will be considered in future works.

It is noted that the $a$-IGZO TFTs here show small $S$ values, $0.224 \mathrm{~V} /$ decade for the depletion-type TFT and $0.104 \mathrm{~V} /$ decade for the enhancement-type TFT (Fig. 1). The subthreshold swing can be expressed as $S$ $=\left(d \log I_{\mathrm{DS}} / d V_{G}\right)^{-1}=2.3 k_{B} T / e\left(1+C_{\mathrm{sg}} / C_{\mathrm{OX}}\right),{ }^{17}$ where $k_{B}$ de- notes the Boltzmann constant, $T$ the temperature, $e$ the elementary electric charge, $C_{\mathrm{OX}}$ the gate insulator capacitance, and $C_{\mathrm{sg}}$ the equivalent capacitance contributed by the subgap states (both bulk and interface). In the current model, effects of the trap states at the gate-insulator interface are implicitly included in bulk subgap states and $C_{\mathrm{sg}}$. From the experimental $S$ values, one calculates the area subgap DOSs of 6.0 $\times 10^{11}$ and $1.6 \times 10^{11} \mathrm{~cm}^{-2} \mathrm{eV}^{-1}$ for the depletion-type TFT and the enhancement-type TFT, respectively. From the simulations, it is known that in the subthreshold regime, the Fermi level is around $E_{C^{-}} 0.5 \mathrm{eV}$. At this energy level, subgap DOSs are $1.0 \times 10^{17}$ and $3.3 \times 10^{16} \mathrm{~cm}^{-3} \mathrm{eV}^{-1}$ for the depletion-type TFT and the enhancement-type TFT, respectively. These numbers correspond to area densities of 4.0 $\times 10^{11}$ and $1.3 \times 10^{11} \mathrm{~cm}^{-2} \mathrm{eV}^{-1}$, considering the $a$-IGZO thickness of $40 \mathrm{~nm}$, roughly consistent with those calculated from experimental $S$ values.

In summary, the characteristics of $a-\mathrm{InGaZnO}_{4}$ TFTs can be well reproduced by a simple model considering a constant mobility (i.e., no electric-field dependence) and a subgap DOS consisting of both tail states and deep gap states. This simple model is useful for both depletion-type and enhancement-type $a$-IGZO TFTs. Results show that the extracted subgap DOSs of $a$-IGZO are orders of magnitude lower than those in amorphous covalent semiconductors such as amorphous silicon. The TFT model presented in this work shall be of general use for designs of TFT devices and circuits from $a$-IGZO or other AOSs and shall benefit further development of the oxide TFT technology toward the computer-aided-design stage.

H.-H. Hsieh would like to acknowledge personal funding from Interchange Association, Japan and National Science Council, Taiwan. T. Kamiya is supported by Industrial Technology Research Grant Program (06A12203d) from NEDO of Japan.

${ }^{1}$ A. Nathan, A. Kumar, K. Sakariya, P. Servati, S. Sambandan, and D. Striakhilev, IEEE J. Solid-State Circuits 39, 1477 (2004).

${ }^{2}$ S. Y. Myong and K. S. Lim, Appl. Phys. Lett. 88, 243510 (2006)

${ }^{3}$ S.-J. Lee, S.-G. Yoon, K.-J. Choi, S.-O. Ryu, S.-M. Yoon, N.-Y. Lee, and B.-G. Yu, J. Vac. Sci. Technol. B 24, 2312 (2006).

${ }^{4}$ K. Nomura, H. Ohta, A. Takagi, T. Kamiya, M. Hirano, and H. Hosono, Nature (London) 432, 488 (2004).

${ }^{5}$ E. Fortunato, P. Barquinha, A. Pimentel, L. Pereira, G. Goncalves, and R. Martins, Phys. Status Solidi (RRL) 1, R34 (2007).

${ }^{6}$ H. Q. Chiang, J. F. Wager, R. L. Hoffman, J. Jeong, and D. A. Keszler, Appl. Phys. Lett. 86, 013503 (2005).

${ }^{7}$ H.-H. Hsieh and C.-C. Wu, Appl. Phys. Lett. 91, 013502 (2007).

${ }^{8}$ Y.-L. Wang, F. Ren, W. Lim, D. P. Norton, S. J. Pearton, I. I. Kravchenko, and J. M. Zavada, Appl. Phys. Lett. 90, 232103 (2007).

${ }^{9}$ H. Hosono, J. Non-Cryst. Solids 352, 851 (2006).

${ }^{10}$ A. Takagi, K. Nomura, H. Ohta, H. Yanagi, T. Kamiya, M. Hirano, and H. Hosono, Thin Solid Films 486, 38 (2005).

${ }^{11}$ K. Nomura, T. Kamiya, H. Ohta, T. Uruga, M. Hirano, and H. Hosono, Phys. Rev. B 75, 035212 (2007).

${ }^{12}$ S. K. O'Leary, J. Mater. Sci.: Mater. Electron. 15, 401 (2004).

${ }^{13}$ M. L. Benkhedir, M. Brinza, and G. J. Adriaenssens, J. Phys.: Condens. Matter 16, S5253 (2004).

${ }^{14}$ O. Tal, Y. Rosenwaks, Y. Preezant, N. Tessler, C. K. Chan, and A. Kahn, Phys. Rev. Lett. 95, 256405 (2005).

${ }^{15}$ ATLAS User's Manual (Silvaco International, Santa Clara, California, 2007).

${ }^{16}$ K. Nomura, A. Takagi, T. Kamiya, H. Ohta, M. Hirano, and H. Hosono, Jpn. J. Appl. Phys., Part 1 45, 4303 (2006).

${ }^{17}$ C. R. Kagan and P. Andry, Thin-Film Transistors (Marcel Dekker, New York, 2003), p. 87. 